\title{
The serum estradiol/oocyte ratio in patients with breast cancer undergoing ovarian stimulation with letrozole and gonadotropins
}

\author{
Yoo Jin Shim ${ }^{1}$, Aeran Seol', Dayong Lee ${ }^{2,3}$, Seul Ki Kim², ${ }^{2,3}$ Jung Ryeol Lee ${ }^{2,3}$, Byung Chul Jee ${ }^{2,3}$, \\ Chang Suk Suh ${ }^{1,3}$, Seok Hyun Kim ${ }^{1,3}$ \\ Department of Obstetrics and Gynecology, ${ }^{1}$ Seoul National University Hospital, Seoul, ${ }^{2}$ Seoul National University Bundang Hospital, Seongnam, ${ }^{3}$ Seoul \\ National University College of Medicine, Seoul, Korea
}

\section{Objective}

To investigate the patterns of estradiol-oocyte ratio (EOR) and estradiol-mature oocyte ratio (EMOR) in patients with breast cancer undergoing controlled ovarian stimulation (COS) using letrozole and gonadotropins for fertility preservation.

\section{Methods}

This retrospective study included 32 breast cancer patients underwent COS for fertility preservation and no patients had previously undergone gonadotoxic treatments. Basal characteristics and in vitro fertilization (IVF) outcomes were compared according to the age of women (age $<35$ vs. age $\geq 35$ ) and the starting phase of ovarian stimulation (early follicular phase vs. late follicular phase vs. luteal phase).

\section{Results}

Patients who were administered with a letrozole-combined regimen, the peak serum estradiol (E2) was maintained at a low level $(386.3 \pm 346.9 \mathrm{pg} / \mathrm{mL})$. EOR was $52.4 \pm 78.8 \mathrm{pg} / \mathrm{mL}$, and EMOR was $71.0 \pm 41.2 \mathrm{pg} / \mathrm{mL}$. When the 2 groups were compared according to the age of women (age <35 vs. age $\geq 35$ ), EOR was $34.5(22.2-46.5) \mathrm{pg} / \mathrm{mL}$ and 40.7 (19.3-65.3) pg/mL, respectively; EMOR was 79.8 (40.8-90.6) pg/mL and 68.8 (44.5-85.9) pg/mL, respectively. There was no significant difference in the IVF outcomes. When the 2 groups were compared according to the starting phase of ovarian stimulation, there were no significant differences in IVF outcomes, EOR and EMOR among the groups.

\section{Conclusion}

Measuring the peak E2 concentration in breast cancer patients undergoing IVF for fertility preservation with a cotreatment of letrozole allows for the prediction of the numbers of oocytes and mature oocytes.

Keywords: Breast neoplasms; Fertility preservation; Aromatase inhibitors; Estradiol; In vitro fertilization

\section{Introduction}

The intensity of ovarian reactivity to controlled ovarian stimulation (COS) is assessed on the basis of serum estradiol (E2) level and follicular diameter, as measured by an ultrasound. It is common to continuously administer gonadotropin until at least 2 follicles reach a mean diameter of $\geq 17-18 \mathrm{~mm}$ and additional ovarian follicles are 14-16 $\mathrm{mm}$ in diameter and until the serum E2 level reflects the overall size and maturity of the ovarian follicles. Follicles with a size of more than $14 \mathrm{~mm}$ has a serum E2 concentration of approximately $200 \mathrm{pg} / \mathrm{mL}$
Received: 2017.06.22. Revised: 2017.08.07. Accepted: 2017.08.21. Corresponding author: Seul Ki Kim

Department of Obstetrics and Gynecology, Seoul National University Bundang Hospital, 82 Gumi-ro 173-beon-gil, Bundanggu, Seongnam 13620, Korea

E-mail: skkim@snubh.org

https://orcid.org/0000-0002-1647-6711

Articles published in Obstet Gynecol Sci are open-access, distributed under the terms of the Creative Commons Attribution Non-Commercial License (http://creativecommons. org/licenses/by-nc/3.0/) which permits unrestricted non-commercial use, distribution, and reproduction in any medium, provided the original work is properly cited.

Copyright $\odot 2018$ Korean Society of Obstetrics and Gynecology 


\section{Obstetrics \& Gynecology Science}

Yoo Jin Shim, et al. The serum estradiol/oocyte ratio

[1]. Therefore, checking the serum E2 level on triggering day helps to predict the number of oocytes retrieved.

During COS for in vitro fertilization (IVF), it is concerning that the serum E2 level may increase to the supraphysiologic level, since such an increase would negatively affect estrogen-dependent cancer, such as breast cancer. For this reason, gonadotropin and aromatase inhibitor (Al) are administered simultaneously to suppress an increase in the E2 level during the course of IVF [2]. Al prevents estrogen production by competitive, reversible binding of Al to the heme of cytochrome P450 unit of aromatase. Tamoxifen or Al is commonly used because they are relatively safe. Azim et al. [3] demonstrated there was no positive correlation between the number of oocytes retrieved and recurrence rate in breast cancer.

There have been studies measuring the total serum E2 in letrozole co-treatment cycles [4]. These studies have shown that an administration of letrozole keeps the E2 concentration low in breast cancer patients during the course of COS. Nevertheless, there has been a paucity of data analyzing relationship between E2 concentration and IVF outcomes. Therefore, the number of oocytes retrieved prior to ovum pick up in letrozole co-treatment cycles was hard to predict. For the group with letrozole-combined administration, analyzing estradiol-oocyte ratio (EOR) will help predict the number of oocytes retrieved and IVF outcomes.

The aim of this study was to investigate the patterns of EOR and estradiol-mature oocyte ratio (EMOR) in patients with breast cancer undergoing COS using letrozole and gonadotropins for fertility preservation.

\section{Materials and methods}

\section{Study subjects and design}

This retrospective study included breast cancer patients undergoing an IVF for fertility preservation between September 2012 and February 2017 in our institution. During the study period, 37 women underwent COS for fertility preservation and no patients had previously undergone gonadotoxic treatments. The exclusion criteria were as follows: patients with known poor ovarian response (POR) according to the Bologna criteria, polycystic ovarian syndrome (PCOS) according to the Rotterdam criteria, endometriosis, or other medical disorders that could potentially affect folliculogenesis. This study was approved by the Institutional Review Board of Seoul National University Bundang Hospital (B-1706-405-101).

\section{Clinical management of cancer patients for fertility preservation}

All patients were evaluated for infertility risk, based on their age and gonadotoxic treatment regimen. They were counseled by an attending reproductive endocrinologist regarding various fertility preserving strategies. Pelvis ultrasound and hormonal profiles, including follicle-stimulating hormone $(\mathrm{FSH})$, luteinizing hormone, E2, progesterone (P4), and antiMullerian hormone (AMH) were evaluated. The menstrual cycle phase was evaluated based on the onset of the last menstrual period, ultrasonographic findings, and serum P4 concentration. The early follicular phase was defined as within the first 5 days of the menstrual cycle, and the late follicular phase was defined as the period of menstrual cycle after the first 5 days with the emergence of a dominant follicle and a serum P4 level of $<3 \mathrm{ng} / \mathrm{mL}$. The luteal phase was determined by a serum P4 level of $\geq 3 \mathrm{ng} / \mathrm{mL}$. In these patients, ovarian stimulation was started immediately, irrespective of their menstrual cycle date, without awaiting menstruation.

\section{COS protocols}

After the baseline pelvic ultrasound and hormonal evaluation, ovarian stimulation with gonadotropin-releasing hormone $(\mathrm{GnRH})$ antagonist protocol was started with recombinant FSH (Gonal-F; Serono, Geneva, Switzerland). The initial dose of gonadotropins was determined based on age and an assessment of ovarian reserve, as estimated by antral follicle count (AFC) and/or AMH level. An Al, letrozole (Femara, 5 mg/day; Novartis, Suffern, NY, USA), was used concomitantly with recombinant FSH until the day of triggering, and then continued again for a week after oocyte retrieval. GnRH antagonist (Cetrorelix, 0.25 mg; Serono) was added to prevent premature ovulation when the lead follicle reached a mean diameter of $14 \mathrm{~mm}$. When at least 2 follicles reached a mean diameter of $\geq 18 \mathrm{~mm}$, the final oocyte maturation was achieved with recombinant human chorionic gonadotropin (hCG; Ovidrel, 250 or $500 \mu \mathrm{g}$; Serono).

Oocyte retrieval was performed 36 hours after hCG triggering, using a transvaginal ultrasound guidance. Oocyte maturity was evaluated by microscopic examination and graded as mature (metaphase II), intermature (metaphase I), and immature. Mature oocytes and in vitro-matured oocytes were 


\title{
Obstetrics \& Gynecology Science
}

\author{
Vol. 61, No. 2, 2018
}

cryopreserved. For embryo banking, intracytoplasmic sperm injection (ICSI) was performed using ejaculated sperm. None of the male partners had subfertile sperm parameters. Fertilization was assessed 16 to 18 hours after ICSI via the presence of 2 pronuclei and a second polar body. The quality of embryos was evaluated in accordance with the morphological criteria based on the fragmentation degree and the regularity of blastomeres on the third day after fertilization. All embryos were cryopreserved on the third day.

\section{Statistical analysis}

Data was analyzed using R software version 2.14.2 (R Foundation, Vienna, Austria; http://www.rproject.org) and compared by Mann-Whitney U, Kruskal-Wallis, or Fisher's exact tests, as indicated. Descriptive data is expressed as the median and range. The result was considered significant when the $P$ value was less than 0.05 .

\section{Results}

During the study interval, oocyte retrievals were performed for 37 breast cancer patients. EOR was $50.4 \pm 75.0 \mathrm{pg} / \mathrm{mL}$, and EMOR was $65.6 \pm 41.9 \mathrm{pg} / \mathrm{mL}$.
To include only the data from patients who had normal ovarian response, patients with POR $(n=2)$ and PCOS $(n=3)$ were excluded from analysis. The mean age of participants was $33.2 \pm 4.8$ years. Oocyte and embryo cryopreservation were performed in 21 and 8 cycles, respectively.

As expected, patients who were administered with a letrozole-combined regimen, the peak serum E2 was maintained at a low level $(386.3 \pm 346.9 \mathrm{pg} / \mathrm{mL})$. The levels of serum E2 were positively correlated with the total number of oocytes retrieved $(r=0.34 ; P=0.06)$ and mature oocytes retrieved $(r=0.49 ; P=0.01)$. EOR was $52.4 \pm 78.8 \mathrm{pg} / \mathrm{mL}$, and EMOR was $71.0 \pm 41.2 \mathrm{pg} / \mathrm{mL}$.

As shown in Table 1, patients were dived into 2 groups by age (age $<35$ vs. age $\geq 35$ ). According to this categorization, there was no significant difference in basal characteristics or stimulation parameters. EOR was 34.5 (22.2-46.5) and 40.7 (19.3-65.3), respectively; EMOR was 79.8 (40.8-90.6) and 68.8 (44.5-85.9), respectively. There was no significant difference in the COS outcomes.

Table 2 compares the baseline characteristics, stimulation parameters, and outcomes according to the starting phase of ovarian stimulation. Ovarian stimulation was started in the early follicular $(n=18)$, late follicular $(n=4)$, and luteal phases $(n=10)$. There were no significant differences with respect to

Table 1. Basal characteristics of the patients and in vitro fertilization outcomes according to patients' age

\begin{tabular}{|c|c|c|c|c|}
\hline Characteristics & Age $<35(n=19)$ & Age $\geq 35(n=13)$ & Total $(n=32)$ & $P$-value \\
\hline Woman's age (yr) & $31.0(28.5-32.5)$ & $38.0(36.0-39.0)$ & $34.0(29.5-36.5)$ & $<0.001$ \\
\hline $\operatorname{BMI}\left(\mathrm{kg} / \mathrm{m}^{2}\right)$ & $22.2(21.5-24.0)$ & $21.1(19.6-22.9)$ & $21.8(20.4-23.7)$ & NS \\
\hline Basal serum FSH (mlU/mL) & $4.8(4.1-5.8)$ & $4.7(2.8-5.2)$ & $4.8(3.6-5.7)$ & NS \\
\hline Basal serum AMH (ng/mL) & $3.0(1.2-4.3)$ & $3.6(1.6-4.8)$ & $3.3(1.5-4.6)$ & NS \\
\hline Total FSH administered (IU) & $2,025.0(1,612.5-2,362.5)$ & $1,800.0(1,800.0-2,400.0)$ & $1,987.5(1,612.5-2,437.5)$ & NS \\
\hline Total stimulation days & $9.0(7.5-10.0)$ & $8.0(7.0-9.0)$ & $9.0(7.0-10.0)$ & NS \\
\hline E2 on day of trigger $(\mathrm{pg} / \mathrm{mL})$ & $324.0(185.0-607.0)$ & $274.0(171.0-366.0)$ & $277.0(177.5-515.0)$ & NS \\
\hline No. of oocytes retrieved & $10.0(4.0-16.5)$ & $8.0(6.0-12.0)$ & $9.0(4.5-14.5)$ & NS \\
\hline No. of mature oocytes retrieved & $4.0(1.5-8.0)$ & $4.0(3.0-5.0)$ & $4.0(2.0-7.0)$ & NS \\
\hline EOR & $34.5(22.2-46.5)$ & $40.7(19.3-65.3)$ & $35.7(20.0-57.0)$ & NS \\
\hline EMOR & $79.8(40.8-90.6)$ & $68.8(44.5-85.9)$ & $70.5(40.8-85.9)$ & NS \\
\hline $\begin{array}{l}\text { No. of mature oocytes } \\
\text { cryopreserved }(n=21)\end{array}$ & $4.5(3.0-9.0)$ & $5.0(4.0-9.5)$ & $5.0(3.0-9.0)$ & NS \\
\hline No. of embryos cryopreserved $(n=8)$ & $8.0(7.0-10.0)$ & $4.5(4.0-5.0)$ & $5.0(4.0-7.5)$ & NS \\
\hline
\end{tabular}

Data are presented as median (interquartile range). Data are compared by Kruskal Wallis analysis.

BMI, body mass index; FSH, follicle-stimulating hormone; AMH, anti-Mullerian hormone; E2, estradiol; EOR, estradiol-oocyte ratio; EMOR, estradiol-mature oocyte ratio; NS, not significant. 


\section{Obstetrics \& Gynecology Science}

Yoo Jin Shim, et al. The serum estradiol/oocyte ratio

Table 2. Basal characteristics of the patients and in vitro fertilization outcomes according to starting phase

\begin{tabular}{|c|c|c|c|c|}
\hline Characteristics & $\begin{array}{l}\text { Early follicular phase } \\
\qquad(n=18)\end{array}$ & $\begin{array}{l}\text { Late follicular phase } \\
\qquad(n=4)\end{array}$ & $\begin{array}{l}\text { Luteal phase } \\
\qquad(n=10)\end{array}$ & $P$-value \\
\hline Woman's age (yr) & $33.5(32.0-36.0)$ & $35.0(31.5-39.0)$ & $34.5(29.0-39.0)$ & NS \\
\hline $\mathrm{BMI}\left(\mathrm{kg} / \mathrm{m}^{2}\right)$ & $21.8(21.0-23.7)$ & $21.1(19.2-23.3)$ & $22.5(19.8-23.7)$ & NS \\
\hline Basal serum FSH (mIU/mL) & $4.9(4.5-5.7)$ & $5.0(3.6-6.4)$ & $3.6(1.5-5.2)$ & NS \\
\hline Basal serum AMH (ng/mL) & $2.7(1.2-4.8)$ & $2.9(1.2-4.7)$ & $3.5(1.8-4.1)$ & NS \\
\hline Total FSH administered (IU) & $1,687.5(1,500.0-2,025.0)^{a)}$ & $2,250.0(1,875.0-2,775.0)^{b)}$ & $2,250.0(2,025.0-2,700.0)^{b)}$ & 0.008 \\
\hline Total stimulation days & $7.5(7.0-9.0)$ & $9.0(8.0-11.5)$ & $9.5(9.0-11.0)$ & 0.008 \\
\hline E2 on day of trigger $(\mathrm{pg} / \mathrm{mL})$ & $324.0(244.0-607.0)^{a)}$ & $138.0(136.5-790.5)^{b)}$ & $209.5(171.0-392.0)^{b)}$ & NS \\
\hline No. of oocytes retrieved & $10.0(5.0-17.0)$ & $8.5(4.0-16.0)$ & $7.0(4.0-12.0)$ & NS \\
\hline No. of mature oocytes retrieved & $4.0(2.0-8.0)$ & $3.5(1.0-10.0)$ & $5.0(2.0-6.0)$ & NS \\
\hline EOR & $38.2(27.3-55.4)$ & $19.3(16.5-42.4)$ & $36.0(15.3-58.5)$ & NS \\
\hline EMOR & $82.0(46.0-91.5)$ & $69.0(48.0-82.6)$ & $57.5(31.6-75.0)$ & NS \\
\hline $\begin{array}{l}\text { No. of mature oocytes } \\
\text { cryopreserved }(n=21)\end{array}$ & $5.0(3.0-9.0)$ & $10.0(5.0-15.0)$ & $4.0(2.0-5.0)$ & NS \\
\hline $\begin{array}{l}\text { No. of embryos cryopreserved } \\
(n=8)\end{array}$ & $7.0(4.0-8.0)$ & $2.5(1.0-4.0)$ & $6.0(5.0-9.0)$ & NS \\
\hline
\end{tabular}

Data are presented as median (interquartile range). Data are compared by Kruskal Wallis analysis.

BMI, body mass index; FSH, follicle-stimulating hormone; AMH, anti-Mullerian hormone; E2, estradiol; EOR, estradiol-oocyte ratio; EMOR, estradiol-mature oocyte ratio; NS, not significant.

${ }^{a, b)}$ Different superscript means a statistical significance within the same row.

age, body mass index, basal serum FSH, and AMH levels. The total gonadotropin dose was significantly higher in the late follicular and luteal phase-start cycles than in early follicular phase-start cycles owing to a longer stimulation. The number of total and mature oocytes retrieved and the oocyte maturity rates were similar irrespective of the start phase of ovarian stimulation. There were also no significant differences in EOR and EMOR among the groups.

\section{Discussion}

Our study is the first study to analyze EOR in breast cancer patients undergoing IVF for fertility preservation with a cotreatment of letrozole. The present study indicated that the levels of serum E2 were positively correlated with the total number of oocytes retrieved and mature oocytes retrieved. We showed that the EOR in these patients was $52.4 \pm 78.8 \mathrm{pg} / \mathrm{mL}$, which was significantly lower than that during the conventional COS cycles. By using the result of this study, E2 level can be used to assess the response to the ovarian stimulation and predict the number of oocytes retrieved in advance of the oocyte pickup in breast cancer patients.

The number of retrieved mature oocytes is more closely related to the IVF outcomes than the total number of retrieved oocytes. Therefore, EMOR can provide additional information compared with EOR. Again, our study is the first study to analyze EMOR in breast cancer patients undergoing IVF for fertility preservation, with a co-treatment of letrozole. The median value of EMOR was $71.0 \pm 41.2 \mathrm{pg} / \mathrm{mL}$ in these patients, which was about 1.35 times higher than that of EOR.

Although the total peak serum E2 level does not have a positive or negative effect on IVF outcomes, EOR and EMOR showed to have an impact on the clinical pregnancy rate. Loumaye et al. [5] first found that EOR may be a reliable indicator for the success of IVF. According to a previous study [6], evaluating the data from over 9,000 IVF cycles, the group with an EOR between 250 and 750 showed a clinically significant increase in the clinical pregnancy rate compared with the group that had an EOR of over 1,500 (odds ratio, 3.4; $95 \%$ confidence interval, 2.67-4.34). They concluded that EOR can be a good predictor of reproductive outcomes. Hence, we 


\section{Obstetrics \& Gynecology Science}

Vol. 61, No. 2, 2018

can assert that EOR and EMOR can be used as predictive factors for reproductive outcomes. As such, a study that analyzes EOR and EMOR may be important.

A future study of pregnancy trial using oocyte cryopreservation or embryo cryopreservation could reveal a correlation between EOR, EMOR, and clinical pregnancy rate in breast cancer patients undergoing IVF for fertility preservation with a co-treatment of letrozole. Further studies are necessary to better enable clinicians to predict IVF outcome by measuring EOR and EMOR in breast cancer patients for the conventional COS groups.

The limitation of this research is that the relation between EOR, EMOR in breast cancer patients and reproductive outcome was not be proved. In the future, if the number of patients attempting to conceive using frozen oocytes increases, the correlation with the pregnancy rate will be analyzed. We hope to set a good precedent for many upcoming papers. Another limitation is that the number of patients analyzed is relatively small. Our academic community needs forwardlooking studies, including a larger number of patients in the future.

In conclusion, measuring the peak E2 concentration in breast cancer patients undergoing IVF for fertility preservation with a co-treatment of letrozole allows for the prediction of the numbers of oocytes and mature oocytes.

\section{Acknowledgements}

This work was supported by the National Research Foundation of Korea (NRF) grant funded by the Korea government (MSIP) (No. NRF-2017R1C1B2003897).

\section{Conflict of Interest}

No potential conflict of interest relevant to this article was reported.

\section{References}

1. Orvieto R, Hod E, Volodarsky M, Homburg R, Rabinson J, Meltcer $S$. Controlled ovarian hyperstimulation: are we monitoring the appropriate sex-steroid hormones? Fertil Steril 2008;89:1269-72.

2. Oktay K, Hourvitz A, Sahin G, Oktem O, Safro B, Cil A, et al. Letrozole reduces estrogen and gonadotropin exposure in women with breast cancer undergoing ovarian stimulation before chemotherapy. J Clin Endocrinol Metab 2006;91:3885-90.

3. Azim AA, Costantini-Ferrando M, Oktay K. Safety of fertility preservation by ovarian stimulation with letrozole and gonadotropins in patients with breast cancer: a prospective controlled study. J Clin Oncol 2008;26:2630-5.

4. Kim JH, Kim SK, Lee HJ, Lee JR, Jee BC, Suh CS, et al. Efficacy of random-start controlled ovarian stimulation in cancer patients. J Korean Med Sci 2015;30:290-5.

5. Loumaye E, Engrand P, Howles CM, O'Dea L. Assessment of the role of serum luteinizing hormone and estradiol response to follicle-stimulating hormone on in vitro fertilization treatment outcome. Fertil Steril 1997;67:889-99.

6. Vaughan DA, Harrity C, Sills ES, Mocanu EV. Serum estradiol:oocyte ratio as a predictor of reproductive outcome: an analysis of data from >9000 IVF cycles in the Republic of Ireland. J Assist Reprod Genet 2016;33:481-8. 